\title{
Periodic Orbits in the Photogravitational Elliptic Restricted Three-Body Problem
}

\author{
Y. SHARON RUTH, RAM KRISHAN SHARMA \\ Department of Aerospace Engineering, Karunya Institute of Technology and Sciences, Coimbatore, 641114, India. \\ Email: ysruth@gmail.com, ramkrishansharma@gmail.com
}

\begin{abstract}
Periodic orbits in the elliptic restricted three-body problem are studied by considering the photogravitational and oblateness effects of the larger and smaller primary, respectively. The mean motion is derived with the help of averaging the distance $r$ between the primaries over a revolution in terms of the mean anomaly. Collinear points L1, L2, L3 are studied for some of the Sun and its planet systems. The value of the critical mass $\mu \mathrm{c}$ is found, which decreases with the increase in radiation pressure and oblateness. The stability of the triangular points is studied using the analytical technique of Bennett. This is based on Floquet's theory for determination of characteristic exponents for periodic coefficients. Transition curves bounding the regions of stability in the $\mu$-e plane, accurate to $\mathrm{O}\left(\mathrm{e}^{2}\right)$ are generated. Tadpole orbits, a combination of long-short periodic orbits, are produced for Sun-Jupiter and Sun-Saturn systems.
\end{abstract}

Keywords: The elliptic restricted three-body problem, radiation pressure, oblateness, transition curve, tadpole orbits.

\section{Introduction}

The elliptic restricted three-body problem (ER3BP) determines the motion of infinestimal body under the gravitational attraction of two finite bodies known as primaries, which revolve in elliptic orbits around their center of mass. The term "restricted" means that the third body gets influenced by the primaries whereas it does not affect their motion. It describes the dynamical system more accurately as the motion of the primaries are mostly elliptical. The photogravitational circular restricted three-body problem (PCR3BP) studied by Radzievsky (1950) is the simplest model for this problem. Poynting (1903) explained that small meteors or cosmic dust are not affected by gravitational force alone but by radiation factor as they come near a luminous body.

Several studies in the perturbed elliptic restricted three-body problem (PER3BP) have been carried out. Some of them are by Raheem and Singh (2006), Kumar and Ishwar (2011), Singh and Umar (2012), etc. It is understood that the rotation of planets produces an equatorial bulge due to centrifugal force; resulting in an oblate spheroid. Some of the planets in the solar system such as Jupiter, Saturn, Uranus and Neptune are sufficiently oblate. Various authors such as Sahoo and Ishwar (2000), Kumar and Ishwar (2009), Narayan and Kumar (2011), Singh and Umar (2012), Narayan (2014), Umar and Singh (2014) have studied the effect of oblateness in ER3BP by taking one or both primaries as a source of radiation or oblate spheroid.

Some of the authors have calculated the mean motion $n$ and others have used the previous values of this important parameter. Essentially the true anomaly was used for averaging the radial distance $r$ over a revolution. Some of the authors did not explain the method of evaluation. However, some of the authors have ignored the effect of eccentricity on the mean motion. Following Danby (1988, page 346), in this paper we have used the mean anomaly $M$ to average the distance $r$ between the primaries to get its average value. Then we have used this average value in computation of the mean motion $n$ of the primaries. This value of $n$ is different from the mean motion which is derived from the true anomaly in the literature. The characteristic exponents can be analyzed by various perturbation techniques. The present paper uses the analytical technique developed by Bennett (1965) based on Floquet's theory [17] for a system with periodic coefficients. The stability of the third body around the triangular equilibrium points results in the form of transition curves in the $\mu-e$ plane, accurate to $\mathrm{O}\left(\mathrm{e}^{2}\right)$. These curves separate the stable region from the unstable region. The collinear points are generally unstable; whereas the 
triangular points are conditionally stable in the linear analysis. This results in long- and short- orbits around the triangular libration points. Gurfil (2006), Erdi (2009) and Elbaz and Fariz (2016) developed new methods for generating periodic orbits around the libration points.

The present study involves in examining the motion of the third body in the ER3BP when the larger primary is a source of radiation and the smaller primary is an oblate spheroid. Analysis of the stability of the triangular points using the analytical technique with the effect of radiation pressure through generation of transition curves is carried out. Tadpole orbits for Sun-Jupiter system are generated. The motion around $\mathrm{L}_{4}$ is studied for the Sun-Saturn system with and without radiation. The paper is organized in 7 sections. Sec 1. provides introduction; Sec 2. determines the equations of motion; Sec 3. provides the location of collinear libration points; Sec 4. deals with the stability of triangular libration points. In Sec 5. the transition curves are generated; Sec 6. deals with tadpole orbits, and Sec 7. concludes with the results and conclusion.

\section{Equations of Motion}

The planar $\zeta=0$ equations of motion are

$$
\begin{aligned}
& \varepsilon^{\prime \prime}-2 \eta^{\prime \prime}=\frac{1}{1+e \cos f} \Omega_{\varepsilon}, \\
& \eta^{\prime \prime}+2 \varepsilon^{\prime}=\frac{1}{1+e \cos f} \Omega_{\eta} .
\end{aligned}
$$

where $f$ is the true anomaly and $p$ is the semi-latus rectum $a\left(1-e^{2}\right)$ with $a$ being the semi-major axis. The distance between the primaries varies when they move in elliptical orbits.

The force function equation is given as (Sahoo and Ishwar, 2000).

$$
\Omega=\frac{1}{2}\left(\varepsilon^{2}+\eta^{2}\right)+\frac{1}{n^{2}}\left[\frac{(1-\mu) q}{r_{1}}+\frac{\mu}{r_{2}}+\frac{\mu A_{2}}{2 r_{2}^{3}}\right],
$$

where $r_{1}$ and $r_{2}$ are the magnitudes of the position of the spacecraft from the bigger and smaller primary, respectively. $q$ is the mass reduction factor of the larger primary and $A_{2}$ is the oblateness coefficient of the smaller primary. The magnitudes of the position of the spacecraft are given as

$$
\begin{aligned}
& r_{1}^{2}=(\varepsilon-\mu)^{2}+\eta^{2}, \\
& r_{2}^{2}=(\varepsilon-(\mu-1))^{2}+\eta^{2} \\
& A=\frac{A E^{2}-A P^{2}}{5 R^{2}}
\end{aligned}
$$

where $\mathrm{AE}$ is the equatorial radius, $\mathrm{AP}$ is the polar radius and $\mathrm{R}$ is the distance between the two primaries (Sharma and Subba Rao, 1976).

$$
q=1-5.6 \times \frac{10^{-5}}{a \delta} k
$$

where a is the particle radius, $\delta$ is the density, and $\mathrm{k}$ is the solar radiation pressure efficiency factor (Sharma, 1987).

\section{Mean Motion}

Mean motion is the angular speed required for a body to complete one orbit, assuming constant speed in a circular orbit and varies for the elliptic orbit. The mean motion of the primaries is obtained from the mean distance by averaging the distance between the primaries (r) as

Differentiating $\mathrm{M}$ with respect to $\mathrm{E}$,

$$
\begin{aligned}
& \frac{1}{2 \pi} \int_{0}^{2 \pi} r d M \\
& M=E-e \sin E
\end{aligned}
$$




$$
d M=(1-e \cos E) d E
$$

The distance between the primaries in terms of eccentric Anomaly (E) is $r=a(1-e \cos E)$.

The integration is done as:

$$
\begin{aligned}
& \frac{1}{2 \pi} \int_{0}^{2 \pi} a(1-e \cos E)(1-e \cos E) d E=\frac{1}{2 \pi} \int_{0}^{2 \pi} a(1-e \cos E)^{2} d E \\
& =\frac{a}{2 \pi} \int_{0}^{2 \pi}(1-e \cos E)^{2} d E=\frac{a}{2 \pi}\left(e^{2}+2\right) \pi=a\left(1+\frac{e^{2}}{2}\right)
\end{aligned}
$$

Now by balancing the gravitational and centrifugal forces, we get

Rearranging, we get

$$
(1-\mu) \mu\left[1+\frac{3}{2} A_{2}\right]=n^{2} \mu(1-\mu)\left(1+\frac{e^{2}}{2}\right)
$$

$$
n^{2}=\frac{1}{\left(1+\frac{e^{2}}{2}\right)}\left[1+\frac{3 A_{2}}{2}\right]
$$

The mean motion obtained is a function of eccentricity and oblateness. This expression for mean motion $n$ is different than obtained earlier by other investigator.

\section{Computation of Lagrangian Points $\mathrm{L}_{1}, \mathrm{~L}_{2}, \mathrm{~L}_{3}$}

The influence of the effect of radiation pressure and oblateness of the primaries causing the shift in the distances of the Lagrangian points $\mathrm{L}_{1}, \mathrm{~L}_{2}, \mathrm{~L}_{3}$ is studied for some of the Sun and its planetary systems. Considerable difference is found when the larger primary is considered as a source of radiation and smaller primary as an oblate spheroid.

The collinear points are obtained from $\Omega_{\varepsilon}=0, y=0$ as

$$
\varepsilon+\frac{1}{n^{2}}\left(-\frac{(1-\mu)(\varepsilon-\mu) q}{r_{1}^{3}}-\frac{\mu(\varepsilon-(\mu-1))}{r_{2}^{3}}-\frac{3 \mu A_{2}(\varepsilon-(\mu-1))}{2 r_{2}^{5}}\right)=0 .
$$

Case (i) $\mathrm{L}_{1}$ location:

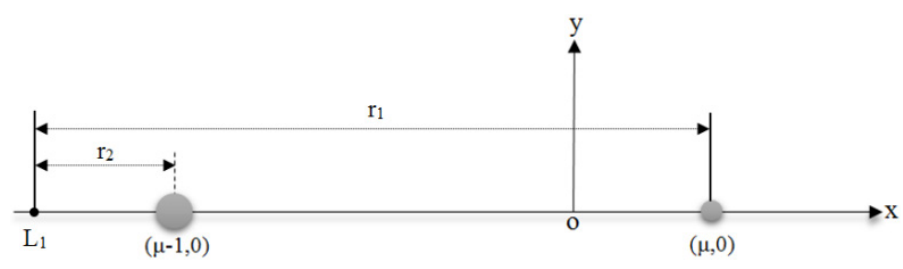

Figure 1. Location of $\mathrm{L}_{1}$ in the $\mathrm{x}-\mathrm{y}$ plane

Substituting $r_{1}=\mu-\varepsilon, r_{2}=\mu-\sigma-\varepsilon$ in Eq. (5) and simplifying, we get

Substituting in the above equation, we get

$$
\varepsilon+\frac{1}{n^{2}}\left(\frac{(1-\mu) q}{(\varepsilon-\mu)^{2}}+\frac{\mu}{(\varepsilon-(\mu-1))^{2}}+\frac{3 \mu A_{2}}{2(\varepsilon-(\mu-1))^{4}}=0\right.
$$

$$
\begin{aligned}
& F(\rho)=2 n^{2} \rho^{7}-\rho^{6}(2 \mu-6) n^{2}-\rho^{5}(4 \mu-6) n^{2}-\rho^{4}(-2 q \mu+2 q+2 \mu) \\
& -\rho^{4}(2 \mu-2) n^{2}-4 \rho^{3} \mu-\rho^{2}\left(2+3 A_{2}\right) \mu-6 \mu A_{2} \rho-3 A_{2} \mu=0
\end{aligned}
$$

Since $\mathrm{F}(0)=-3 A_{2} \mu<0, F(1)=\left(-8 n^{2}+2 q-9 A_{2}-6\right) \mu+18 n^{2}-2 q>0$, according to Descarte's sign rule, a single sign change represents that there is only one positive root for all $0<\mu \leq 1 / 2$. $\rho$ can be 
obtained by finding the roots of the equation using Newton Raphson method and the abscissa of Lagrangian point $\mathrm{L}_{1}$ is calculated from $x=\mu-1-\rho$.

Case (ii) $\mathrm{L}_{2}$ location:

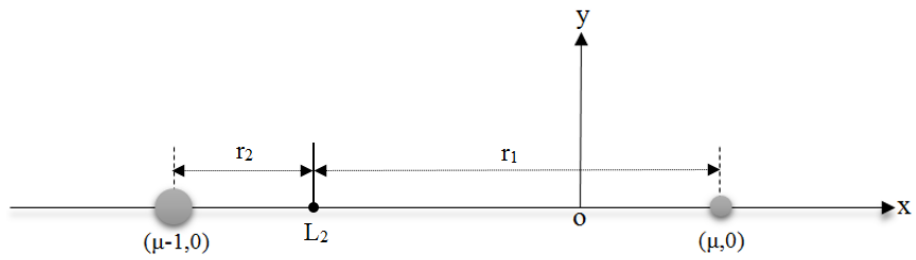

Figure 2. Location of $\mathrm{L}_{2}$ in the $\mathrm{x}-\mathrm{y}$ plane

Substituting $r_{1}=\mu-\varepsilon, r_{2}=1-\mu+\varepsilon$ in Eq.(5) and taking $r_{2}=\rho, r_{1}=1+\rho, \varepsilon=\mu-1+\rho$ in the equation and simplifying, we get

$$
\begin{aligned}
& G(\rho)=2 n^{2} \rho^{7}+n^{2} \rho^{6}(2 \mu-6)+\rho^{5}(6-4 \mu)+n^{2} \rho^{4}(2 \mu-2) \\
& +4 \rho^{3} \mu+\rho^{4}(-2 q \mu-2 \mu+2 q)+\rho^{2}\left(-3 A_{2} \mu-2 \mu\right)+6 A_{2} \mu \rho-3 A_{2} \mu=0
\end{aligned}
$$

Since $\mathrm{G}(0)=-3 A_{2} \mu<0, G(1)=\left(4 n^{2}-2 q-4\right) \mu-6 n^{2}+2 q+6>0$ according to Descartes' sign rule, a single sign change represents that there is only one positive root for all $0<\mu \leq 1 / 2$. $\rho$ can be obtained by finding the roots of the equation using the Newton Raphson method and the abscissa of Lagrangian point $\mathrm{L}_{2}$ is calculated from $x=\mu-1+\rho$.

Case (iii) $\mathrm{L}_{3}$ location:

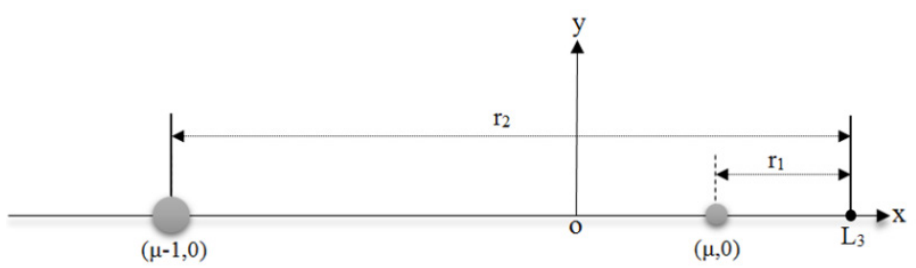

Figure 3. Location of $\mathrm{L}_{3}$ in the $\mathrm{x}-\mathrm{y}$ plane

Substituting $r_{1}=\varepsilon-\mu, r_{2}=1-\mu+\varepsilon$ in Eq.(5) and taking $r_{1}=\rho, r_{2}=1+\rho, \varepsilon=\mu+\rho$ in the equation and simplifying, we get

$$
\begin{aligned}
& H(\rho)=2 n^{2} \rho^{7}+n^{2} \rho^{6}(2 \mu+8)+n^{2} \rho^{5}(8 \mu+12) \\
& +\rho^{4}\left(2 q \mu-2 \mu-2 q+12 \mu n^{2}+8 n^{2}\right)+\rho^{3}\left(8 q \mu-4 \mu-8 q+8 \mu n^{2}+2 n^{2}\right) \\
& +\rho^{2}\left(12 q \mu-3 A_{2} \mu-2 \mu-12 q+2 n^{2} \mu\right)+\rho(8 q \mu-8 q)+2 q \mu-2 q=0
\end{aligned}
$$

Since $H(0)=2 q \mu-2 q<0, H(1)=\left(30 n^{2}+32 q-3 A_{2}-8\right) \mu+34 n^{2}-32 q>0$, according to Descartes' sign rule, a single sign change represents that there is only one positive root. Since this root is near +1 it is advantageous to introduce $\rho=1+\eta$ (page 138, Szebehely) which gives

$$
\begin{aligned}
& H(1+\eta)=2 n^{2} \eta^{7}+\eta^{6}\left(2 n^{2} \mu+22 n^{2}\right)+\eta^{5}\left(20 n^{2} \mu+102 n^{2}\right) \\
& +\eta^{4}\left(2 q \mu+82 n^{2} \mu-2 \mu-2 q+258 n^{2}\right)+\eta^{3}\left(16 q \mu+176 n^{2} \mu-12 \mu-16 q+384 n^{2}\right) \\
& +\eta^{2}\left(48 q \mu+208 n^{2} \mu-3 A_{2} \mu-26 \mu-48 q+336 n^{2}\right)+\eta\left(64 q \mu+128 n^{2} \mu-6 A_{2} \mu-24 \mu-64 q+160 n^{2}\right) \\
& +32 q \mu+32 n^{2} \mu-3 A_{2} \mu-8 \mu+32 n^{2}-32 q=0
\end{aligned}
$$

$\rho$ can be obtained by finding the roots of the above equation using Newton-Raphson method and the abscissa of Lagrangian point $\mathrm{L}_{3}$ is calculated from $x=\mu+\rho$. 
Table 1. Parameters of Sun and its planet systems

\begin{tabular}{llllll}
\hline S.No & Systems & $\mathrm{R}(\mathrm{km})$ & $\mathrm{e}$ & $\mathrm{\mu}$ & \multicolumn{1}{c}{$\mathrm{A}_{2}$} \\
\hline 1 & Sun-Earth & 149600000 & 0.01671022 & $3.0025 \mathrm{E}-06$ & $2.43372 \mathrm{E}-12$ \\
2 & Sun-Mars & 227943824 & 0.09339410 & $3.22605 \mathrm{E}-07$ & $5.21371 \mathrm{E}-13$ \\
3 & Sun-Jupiter & 778340821 & 0.04838624 & 0.000953356 & $2.1183 \mathrm{E}-10$ \\
4 & Sun-Saturn & 1426666422 & 0.05386179 & 0.000285635 & $6.65024 \mathrm{E}-11$ \\
5 & Sun-Uranus & 2870658186 & 0.04725744 & $4.36411 \mathrm{E}-05$ & $7.18673 \mathrm{E}-13$ \\
\hline
\end{tabular}

Table 2. Shifts in the positions of $\mathrm{L}_{1}$ with and without radiation

\begin{tabular}{|c|c|c|c|}
\hline \multirow[b]{2}{*}{ S.No } & \multicolumn{2}{|c|}{$\mathrm{L}_{1}$} & \multirow[b]{2}{*}{$\begin{array}{l}\text { Shift } \\
\text { (in km) }\end{array}$} \\
\hline & $\begin{array}{l}\text { With radiation } \\
(\mathrm{q}=0.9999)\end{array}$ & $\begin{array}{c}\text { Without radiation } \\
(\mathrm{q}=1)\end{array}$ & \\
\hline 1 & -1.010037897 & -1.010032997 & -733.039 \\
\hline 2 & -1.005290777 & -1.004762577 & -120399.92 \\
\hline 3 & -1.068959344 & -1.004762577 & -109901.72 \\
\hline 4 & -1.046232964 & -1.046064464 & -240393.29 \\
\hline 5 & -1.024685758 & -1.024564958 & -346775.50 \\
\hline
\end{tabular}

It is inferred that with the inclusion of radiation pressure, $\mathrm{L}_{1}$ moves away from the larger primary.

Table 3. Shifts in the positions of $\mathrm{L}_{2}$ with and without radiation

\begin{tabular}{|c|c|c|c|}
\hline \multirow[b]{2}{*}{ S.No } & \multicolumn{2}{|r|}{$\mathrm{L}_{2}$} & \multirow{2}{*}{$\begin{array}{l}\text { Shift } \\
(\text { in } \mathrm{km})\end{array}$} \\
\hline & $\begin{array}{c}\text { With radiation } \\
\quad(\mathrm{q}=0.9999) \\
\end{array}$ & $\begin{array}{c}\text { Without radiation } \\
(\mathrm{q}=1)\end{array}$ & \\
\hline 1 & -0.990031597 & -0.990027697 & -583.439 \\
\hline 2 & -0.995675377 & -0.995251877 & -96534.20 \\
\hline 3 & -0.932476443 & -932377943 & -76666.57 \\
\hline 4 & -0.954886565 & -0.954753964 & -189175.9 \\
\hline 5 & -0.975850258 & -0.975745058 & -301993.2 \\
\hline
\end{tabular}

It is inferred that with the inclusion of radiation pressure, $\mathrm{L}_{2}$ moves away from the larger primary.

Table 4. Shifts in the positions of $\mathrm{L}_{3}$ with and without radiation

\begin{tabular}{|c|c|c|c|}
\hline \multirow[b]{2}{*}{ S.No } & \multicolumn{2}{|r|}{$\mathrm{L}_{3}$} & \multirow{2}{*}{$\begin{array}{c}\text { Shift } \\
\text { (in km) }\end{array}$} \\
\hline & $\begin{array}{c}\text { With radiation } \\
(\mathrm{q}=0.9999)\end{array}$ & $\begin{array}{c}\text { Without radiation } \\
\qquad(\mathrm{q}=1)\end{array}$ & \\
\hline 1 & 1.00001440 & 1.00000120 & 1974.719 \\
\hline 2 & 1.00014184 & 1.00000012 & 323292.72 \\
\hline 3 & 1.00075385 & 1.00039725 & 277556.33 \\
\hline 4 & 1.00056893 & 1.00011903 & 641857.22 \\
\hline 5 & 1.00031329 & 1.00001811 & 847300.31 \\
\hline
\end{tabular}

It is inferred that with the inclusion of radiation pressure, $\mathrm{L}_{3}$ moves away from the larger primary. Due to larger distances between the primaries, it is seen that the difference between the locations of $\mathrm{L}_{1}$, $\mathrm{L}_{2}, \mathrm{~L}_{3}$ with and without radiation pressure is also large. The value of $\mathrm{q}$ is kept same $(0.9999)$ in all the computations above. Eccentricity also plays a role in the shifts.

\section{$4 \quad$ Stability of Triangular Points}

We have $\Omega_{\varepsilon}=0, \Omega_{\eta}=0$. Therefore, the gradients of the potential are given by 


$$
\begin{aligned}
& \varepsilon+\frac{1}{n^{2}}\left(-\frac{(1-\mu)(\varepsilon-\mu) q}{r_{1}^{3}}-\frac{\mu(\varepsilon-(\mu-1))}{r_{2}^{3}}-\frac{3 \mu A_{2}(\varepsilon-(\mu-1))}{2 r_{2}^{5}}\right)=0, \\
& \eta\left(1+\frac{1}{n^{2}}\left(-\frac{(1-\mu) q}{r_{1}^{3}}-\frac{\mu}{r_{2}^{3}}-\frac{3 \mu A_{2}}{2 r_{2}^{5}}\right)\right)=0 .
\end{aligned}
$$

Considering $\eta \neq 0$, we arrive with the following equation.

Similarly, for $\mathrm{r}_{2}$

$$
\begin{gathered}
1+\frac{1}{n^{2}}\left(-\frac{q}{r_{1}^{3}}\right)=0 \\
r_{1}^{3}=\frac{q}{n^{2}}
\end{gathered}
$$

$$
\begin{aligned}
& 1+\frac{1}{n^{2}}\left(-\frac{1}{r_{2}^{3}}-\frac{3 A_{2}}{2 r_{2}^{5}}\right)=0 \\
& r_{2}^{3}=\frac{1}{n^{2}}\left[1+\frac{3 A_{2}}{2 r_{2}^{2}}\right]
\end{aligned}
$$

When the primary is considered as a point mass,

$$
r_{2}=n^{-2 / 3}
$$

Considering the oblateness effect,

$$
r_{2}=n^{-2 / 3}+\delta_{2}
$$

Using binomial expansion,

$$
n^{2}=\left[1+\frac{3 A_{2}}{2}+\frac{e^{2}}{2}\right]
$$

If $A_{2}=0$, and neglecting higher order terms, we get

After further substitution, we get

$$
n^{-2 / 3}=\left[1+\frac{e^{2}}{6}\right]
$$

$$
\begin{aligned}
& r_{1}=1+\frac{e^{2}}{6}-\frac{A_{2}}{2}-\frac{\epsilon}{3} \\
& r_{2}=1+\frac{e^{2}}{6}
\end{aligned}
$$

Then the triangular points are obtained as,

$$
\begin{aligned}
& \varepsilon=\mu-\frac{1}{2}-\frac{\epsilon}{3}-\frac{A_{2}}{2} \\
& \eta= \pm \frac{\sqrt{3}}{2}\left[1-\frac{2 \epsilon}{9}-\frac{A_{2}}{3}\right]
\end{aligned}
$$

If the radiation and oblateness terms are eliminated $\epsilon=A_{2}=0$ and eccentricity $e=0$, then the system reduces to $\mathrm{CR} 3 \mathrm{BP}$ and the location of the triangular equilibrium points are given as $\varepsilon=\frac{1}{2}(2 \mu-1)$ and $\eta= \pm \frac{\sqrt{3}}{2}$

\section{Stability of Triangular Points}

The variational equations of motion [10] are 


$$
\begin{aligned}
& u^{\prime \prime}-2 v^{\prime}=\frac{1}{1+e \cos f}\left(-u \Omega_{\varepsilon \varepsilon}-v \Omega_{\varepsilon \eta}\right) \\
& u^{\prime \prime}+2 v^{\prime}=\frac{1}{1+e \cos f}\left(-u \Omega_{\varepsilon \eta}-v \Omega_{\eta \eta}\right)
\end{aligned}
$$

The characteristic equation is given as

$$
\lambda^{4}+\left(4-\Omega_{\varepsilon \varepsilon}-\Omega_{\eta \eta}\right) \lambda^{2}+\left(\Omega_{\varepsilon \varepsilon} \Omega_{\eta \eta}-\left(\Omega_{\varepsilon \eta}\right)^{2}\right)=0
$$

Eliminating the higher order terms and restricting terms to $e^{2}, \epsilon, A_{2}$, we get

$$
\lambda^{4}+\left(1+e^{2}-2 \epsilon-3 A_{2}\right) \lambda^{2}+\frac{27 \mu}{4}(1-\mu)=0
$$

The stability theorem [7] states that if the condition $\frac{\sqrt{7}}{4} \leq e<1$ is satisfied by $e$ of the orbits of the attracting masses, then the solution (11) is unstable. Hence the proof is provided by taking $\epsilon=A_{2}=0$, putting it into Eq. (11), the roots are found out to be

$$
\lambda= \pm \frac{\sqrt{-1-e^{2} \pm\left(\sqrt{\left(\left(1+e^{2}\right)^{2}\right.}-27 \mu(1-\mu)\right)}}{4}
$$

Taking the inequality,

$$
\frac{-1-e^{2}}{4} \geq 0
$$

The eccentricity does not satisfy the above inequality. Hence, it does not hold when $\frac{\sqrt{7}}{4} \leq e<1$ contain real roots or complex roots. For both the cases there must definitely be negative real parts of roots. This will yield that the Lagrangian solutions in Eq. (11) are stable.

By considering $\lambda^{2}=\Lambda$ in Eq. (11),

$$
\Lambda^{2}+\left(1+e^{2}-2 \epsilon-3 A_{2}\right) \Lambda+\frac{27 \mu}{4}(1-\mu)=0
$$

The critical mass can be obtained from the relation, which is found to be

$$
\mu_{c}=\frac{1}{2}-\frac{\sqrt{69}}{18}+\frac{2 e^{2}}{3 \sqrt{69}}-\frac{4 \epsilon}{3 \sqrt{69}}-\frac{6 A_{2}}{3 \sqrt{69}} .
$$

When $\epsilon=A_{2}=0$ the above mass factor becomes that of the classical elliptic case as [1]

$$
\mu_{c}=\frac{1}{2}-\frac{\sqrt{69}}{18}+\frac{2 e^{2}}{3 \sqrt{69}}+\ldots
$$

The classical elliptical case has the critical mass parameter at $\mu_{c}=0.0385 \ldots$

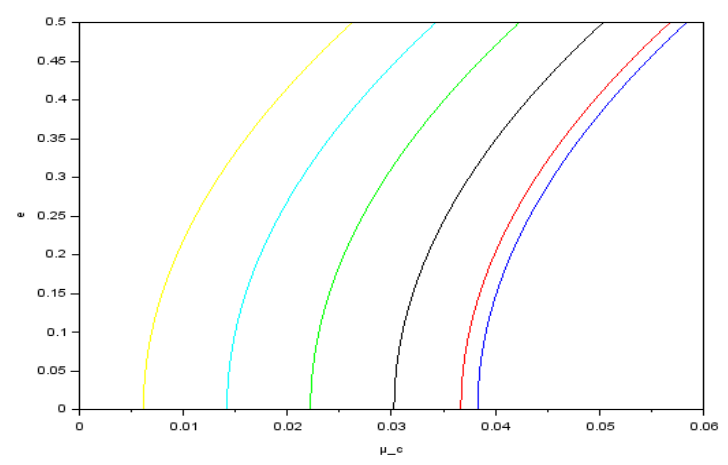

Figure 4. Critical mass vs. Eccentricity 
In Fig.(4) six curves have been plotted taking eccentricity $e$ ( 0 to 0.5$)$, and by considering different values of $\mu(0$ to 0.05$)$. The blue curves indicate the classical ER3BP where the singularity is at $\mu_{c}=0.0385 \ldots$ and this is one of two singularities drawn by [1]. As seen in Fig.(4), the singularity moves towards the left, when the oblateness and radiation pressure of the primaries increase. Indeed, it affects the stability of the triangular points. It is observed that when bodies are oblate and radiating, the stability decreases.

\section{Transition Curve}

Using the analytical method of Bennet(1965), the following relation is determined

$$
X=\left\{\begin{array}{c}
u \\
v \\
u^{\prime} \\
v^{\prime}
\end{array}\right\} \quad X^{\prime}=\left\{\begin{array}{c}
u^{\prime} \\
u^{\prime \prime} \\
v^{\prime} \\
v^{\prime \prime}
\end{array}\right\} \quad p(f, e)=\left(\begin{array}{cccc}
0 & 0 & 1 & 0 \\
0 & 0 & 0 & 1 \\
\phi \Omega_{\varepsilon \varepsilon} & \phi \Omega_{\varepsilon \eta} & 0 & 2 \\
\phi \Omega_{\eta \varepsilon} & \phi \Omega_{\eta \eta} & -2 & 0
\end{array}\right\}
$$

where $\varphi=\frac{1}{1+e \cos f}$

Writing it in matrix notation,

$$
X^{\prime}=p X
$$

Utilizing the Floquet's theory for the periodic coefficients, the form of the solution is

$$
X_{K}=y_{K} e^{\lambda_{k} f}
$$

where $y_{k}$ is the periodic of period T. By discarding the subscript and differentiating the Eq. (16) with respect to $f$ and substituting it in Eq. (15), we get

Expanding $y, \lambda$ and the matrix p into

$$
y^{\prime}=(p-I \lambda) y
$$

$$
\begin{aligned}
& y=y^{0}+y^{(1)} e+y^{(2)} e^{2}+\ldots \\
& \lambda=\lambda_{0}+\lambda_{1} e+\lambda_{2} e^{2}+\ldots \\
& p(f, e)=p^{(0)}+p^{(1)} e+p^{(2)} e^{2}+\ldots
\end{aligned}
$$

where

$$
p(m)=(-\cos f)^{m} \quad c m=1,2,3
$$

and

$$
p^{(0)}=\left(\begin{array}{cccc}
0 & 0 & 1 & 0 \\
0 & 0 & 0 & 1 \\
\Omega_{\varepsilon \varepsilon} & \Omega_{\varepsilon \eta} & 0 & 2 \\
\Omega_{\eta \varepsilon} & \Omega_{\eta \eta} & -2 & 0
\end{array}\right) \quad c=\left(\begin{array}{cccc}
0 & 0 & 0 & 0 \\
0 & 0 & 0 & 0 \\
\Omega_{\varepsilon \varepsilon} & \Omega_{\varepsilon \eta} & 0 & 0 \\
\Omega_{\eta \varepsilon} & \Omega_{\eta \eta} & 0 & 0
\end{array}\right)
$$

Substituting Eq. (18) into Eq. (16), we bring on

$$
\begin{aligned}
& y^{(0)}+y^{(1)} e+y^{(2)} e^{2}+\ldots \\
& =\left\{\left[\left(p^{0}+p^{(1)} e+p^{(2)} e^{2}+\ldots\right)-I\left(\lambda_{0}+\lambda_{1} e+\lambda_{2} e^{2}+\ldots\right)\right]\left(y^{(0)}+y^{(1)} e+y^{(2)} e^{2}+\ldots\right)\right\}
\end{aligned}
$$

The particular solution is given by

$$
y^{(n)}=\sum_{k=-n}^{+n}\left(\begin{array}{c}
a_{1}^{(n, k)} \\
a_{2}^{(n, k)} \\
a_{3}^{(n, k)} \\
a_{4}^{(n, k)}
\end{array}\right) e^{i k f} n=0,1,2, \ldots
$$


If the Zeroth-order solution is assumed to have a constant vector then the non-homogenous terms have frequencies $\leq n / 2 \pi$ for the $\mathrm{n}^{\text {th }}$-order equation.

Equating Eq. (19) according to the coefficient of $e$ and its powers and substituting Eq. (20) in it, we get

$$
\begin{aligned}
& \left(I \lambda_{0}-p^{(0)}\right) a^{(0,0)}=0 \\
& \left(I \lambda_{0}-p^{(0)}\right) a^{(1,0)}=-\lambda_{1} a^{(0,0)} \\
& \left(I\left(\lambda_{0}+i\right)-p^{(0)}\right) a^{(1,+1)}=-\frac{1}{2} c a^{(0,0)} \\
& \left(I\left(\lambda_{0}-I\right)-p^{(0)}\right) a^{(1,-1)}=-\frac{1}{2} c a^{(0,0)} \\
& \left(I \lambda_{0}-p^{0}\right) a^{(2,0)}=-\lambda_{1} a^{(1,0)}+\left(\frac{1}{2} c-I \lambda_{2}\right) a^{(0,0)}-\frac{1}{2} c\left(a^{(1,+1)}+a^{(1,-1)}\right)
\end{aligned}
$$

Since the solution for the elements of $a^{(0,0)} \neq 0$, we have from the first of Eqs. $(21)$

$$
\operatorname{det}\left(I \lambda_{0}-p^{(0)}\right)=0
$$

The characteristic equation is given as

$$
\lambda^{4}+\left(4-\Omega_{\varepsilon \varepsilon}-\Omega_{\eta \eta}\right) \lambda^{2}+\left(\Omega_{\varepsilon \varepsilon} \Omega_{\eta \eta}-\left(\Omega_{\varepsilon \eta}\right)^{2}\right)=0
$$

For the classical CR3BP, the relation can be rewritten as

$$
\lambda_{0}^{4}+\lambda_{0}^{2}+\frac{27}{4} \mu(1-\mu)=0
$$

Taking the determinant of the coefficients on the left of the second of Eq. (21) be zero by the nonhomogenous terms on the right replacing any column of it, then

$$
\operatorname{det}\left(I \lambda_{0}-p^{(0)}\right)+\lambda_{1} a^{(0,0)}=0
$$

As the determinant cannot be zero, we conclude that $\lambda_{1}=0$.

From the third and fourth equations of (24), values of $a^{(1,+1)}$ and $a^{(1,-1)}$ can be calibrated and it is substituted in the last Eq. (21) to yield

$$
\left(I \lambda_{0}-p^{(0)}\right) a^{(2,0)}=\frac{1}{4} c\left\{\left[I\left(\lambda_{0}+i\right)-p^{0}\right]^{-1}+\left[I\left(\lambda_{0}-i\right)-p^{0}\right]^{-1}\right\} c a^{(0,0)}+\left(\frac{1}{2} c-I \lambda_{2}\right) a^{0,0}
$$

In the above equation, considering the real part of any one of the complex conjugate terms on the first part of the equation on the left, we arrive with

$$
\left(I \lambda_{0}-p^{0}\right) a^{(2,0)}=\left\{\frac{1}{2} c R_{e}\left[I\left(\lambda_{0}-i\right)-p^{0}\right]^{-1} c a^{(0,0)}+\left(\frac{1}{2} c-I \lambda_{2}\right)\right\} a^{(0,0)} v
$$

The solution of this system is given by

$$
\lambda=\lambda_{0}+\lambda_{2} e^{2}
$$

When this relation is mathematically executed, $\lambda_{2}$ is of the form:

where

$$
\lambda_{2}=-\left[\frac{\left(Q^{2}-4 R-16\right) \lambda_{0}^{2}+A_{0} F_{0}+A_{1} F_{1}+A_{2} F_{2}}{4\left(Q^{2}-4 Q-4 R\right) \lambda_{0}^{2}+32 R}\right] \lambda_{0}
$$




$$
\begin{aligned}
& A_{0}=\left[(Q+4)^{2}(Q-4)-4 Q R\right] \lambda_{0}^{2}-R(Q+4)-4 R^{2} \\
& A_{1}=-8 \lambda_{0} R\left[2 \lambda_{0}^{2}-(Q+4)\right] \\
& A_{2}=-\lambda_{0}^{2} R\left[Q^{2}-4 R-16\right] \\
& N=\lambda_{0}^{2}\left[4 Q^{2}+8 Q+4-16 R\right]-4 R+(Q+1)^{2} \\
& F_{0}=1 / N\left[\lambda_{0}^{2}(Q+1)+(Q+1)+2 R\right] \\
& F_{1}=-\lambda_{0} / N\left[2 \lambda_{0}^{2}+(Q+3)\right] \\
& F_{2}=-1 / N\left[6 \lambda_{0}^{2}-(Q+1)\right] \\
& Q=-\left(4-\Omega_{\varepsilon \varepsilon}-\Omega_{\eta \eta}\right) \\
& R=\Omega_{\varepsilon \varepsilon} \Omega_{\eta \eta}-\Omega_{\varepsilon \eta}^{2}
\end{aligned}
$$

The transition curve is generated by equating the values of periodic solutions to the expression of characteristic exponents. In the range of interest $0 \leq \mu \leq 1$, the periodic solution produces

$$
\lambda^{*}= \pm \frac{i}{2}
$$

By making $\lambda^{*}=\lambda$, Eq. (23) becomes

$$
-\frac{1}{4}=\left(\lambda_{0}+\lambda_{2} e^{2}\right)^{2}
$$

Eq. (25) generates the transition curve which separates the stable region from the unstable region as shown in Fig. (7) and Fig. (8), where the spacecraft will be stable in the region inside the curve until the singularity $\mu_{c}=0.0385 \ldots$ while the area outside the curve is unstable. The transition curve as shown in Fig. (6) deviates from the numerical results as the eccentricity increases and the radiation of the larger primary affect the stability of the triangular points. The stability decreases when the radiation pressure increases.

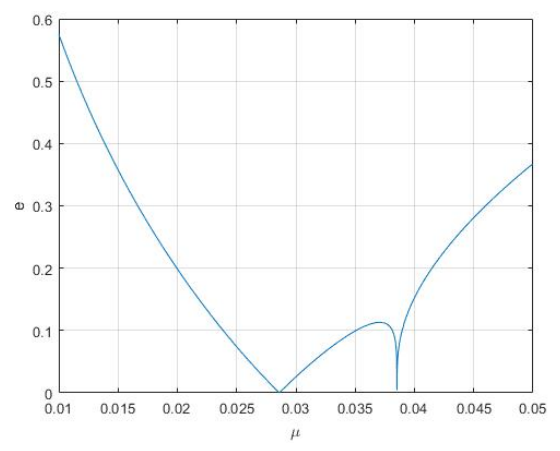

Figure 5. Transition curve when $\in=\mathrm{A}_{2}=0$

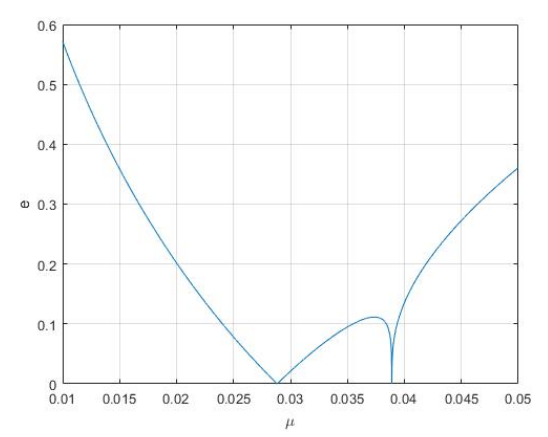

Figure 6. Transition curve when $\epsilon=0.0004, \mathrm{~A}_{2}=0.0002$ 


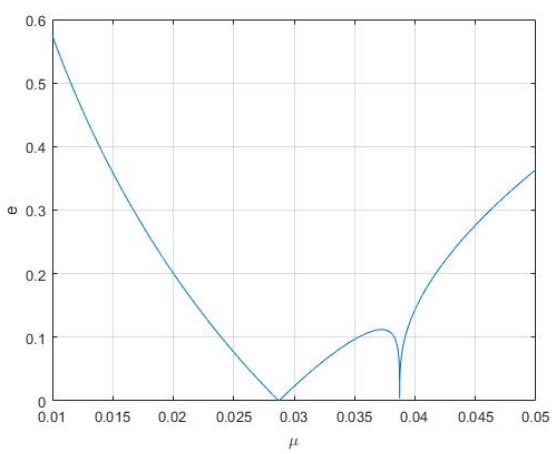

Figure 7. Transition curve when $\epsilon=0.0006, \mathrm{~A}_{2}=0.0004$

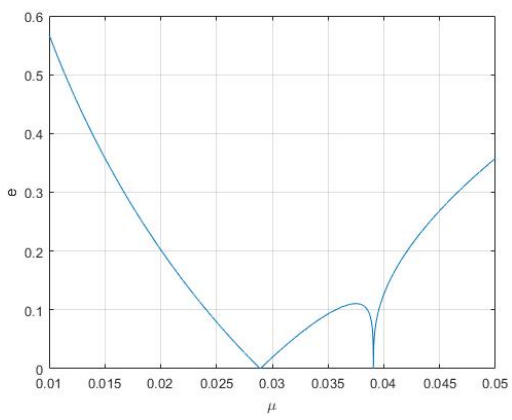

Figure 8. Transition curve when $\epsilon=0.0008, \mathrm{~A}_{2}=0.0006$

\section{$6 \quad$ Tadpole Orbits}

The motion around the triangular libration points are characterized by stable oscillations known as tadpole orbits. They constitute two kinds of orbits: a short-period epicyclical oscillation which is associated with the orbital period of the second primary and a long-period librational motion around an equilibrium point. In this paper, the study focuses on periodic solutions in the vicinity of these triangular libration points, specifically tadpole orbits for Sun-Jupiter system. The perturbational effects due to the radiation pressure of Sun and the oblateness of the Saturn are also studied. We obtain the Tadpole orbits by solving the variational equations of motion with the help of the analytical method.

\section{Periodic orbits around libration points}

For the triangular points, the characteristic equation is given by

$$
\lambda^{4}+\left(4-\frac{3}{\sqrt{\left(1-e^{2}\right)}}\right) \lambda^{2}+\frac{1}{1-e^{2}} \frac{27}{4} \mu(1-\mu)=0
$$

Eigen values for the above equation are obtained as

$$
\lambda^{2}=-\frac{1}{2}\left(4-\frac{3}{\sqrt{1-e^{2}}}\right) \pm \frac{1}{2} \sqrt{\left(4-\frac{3}{\sqrt{1-e^{2}}}\right)^{2}-\frac{27 \mu(1-\mu)}{1-e^{2}}}<0
$$

The generic solution is given as

$$
\begin{aligned}
& x(f)=\alpha_{1} \cos \left(v_{1} f\right)+\alpha_{2} \sin \left(v_{1} f\right)+\alpha_{3} \cos \left(v_{2} f\right)+\alpha_{4} \sin \left(v_{2} f\right) \\
& y(f)=\beta_{1} \cos \left(v_{1} f\right)+\beta_{2} \sin \left(v_{1} f\right)+\beta_{3} \cos \left(v_{2} f\right)+\beta_{4} \sin \left(\vartheta_{2} f\right)
\end{aligned}
$$

Solving the system of variational equations to get the amplitudes $\alpha_{1}, \alpha_{2}, \alpha_{3}, \alpha_{4}$ and $\beta_{1}, \beta_{2}, \beta_{3}, \beta_{4}$, respectively. The initial conditions for the closer motion around $\mathrm{L}_{4}$ are given by $x_{0}=y_{0}=10^{-5}$, $x_{0}^{\prime}=y_{0}^{\prime}=10^{-5}$. Then 


$$
\begin{array}{ll}
\beta_{1}=-\frac{\left(\Omega_{\xi \eta}-2\right) v_{1}^{2}+\Omega_{\xi \xi} \Omega_{\xi \eta}-2 \Omega_{\xi \xi}}{400000 v_{1}^{2}+100000 \Omega_{\xi \eta}^{2}} & \beta_{2}=-\frac{2 v_{1}^{4}+\left(\Omega_{\xi \eta}+2 \Omega_{\xi \xi}\right) v_{1}^{2}+\Omega_{\xi \xi} \Omega_{\eta \eta}}{400000 v_{1}^{3}+100000 \Omega_{\xi \eta}^{2} v_{1}} \\
\beta_{3}=-\frac{\left(\Omega_{\xi \eta}-2\right) v_{2}^{2}+\Omega_{\xi \xi} \Omega_{\xi \eta}-2 \Omega_{\xi \xi}}{400000 v_{2}^{2}+100000 \Omega_{\xi \eta}^{2}} & \beta_{4}=-\frac{\left(2 v_{2}^{4}+\left(\Omega_{\xi \eta}+2 \Omega_{\xi \xi}\right) v_{2}^{2}+\Omega_{\xi \xi} \Omega_{\eta \eta}\right)}{400000 v_{2}^{3}+100000 \Omega_{\xi \eta}^{2} v_{2}} \\
\alpha_{1}=-\frac{\left(\Omega_{\xi \eta}+2\right) v_{1}^{2}+\Omega_{\eta \eta} \Omega_{\xi \eta}+2 \Omega_{\eta \eta}}{400000 v_{1}^{2}+100000 \Omega_{\xi \eta}^{2}} & \alpha_{2}=\frac{2 v_{1}^{4}+\left(2 \Omega_{\eta \eta}-\Omega_{\xi \eta}\right) v_{1}^{2}-\Omega_{\xi \xi} \Omega_{\xi \eta}}{400000 v_{1}^{3}+100000 \Omega_{\xi \eta}^{2} v_{1}} \\
\alpha_{3}=-\frac{\left(\Omega_{\xi \eta}+2\right) v_{2}^{2}+\Omega_{\eta \eta} \Omega_{\xi \eta}+\Omega_{\eta \eta}}{400000 v_{2}^{2}+100000 \Omega_{\xi \eta}^{2}} & \alpha_{4}=\frac{2 v_{2}^{3}+\left(2 \Omega_{\eta \eta}-\Omega_{\xi \eta}\right)-\Omega_{\xi \xi} \Omega_{\xi \eta}}{400000 v_{2}^{3}+100000 \Omega_{\xi \eta}^{2} v_{2}}
\end{array}
$$

The solution for the frequencies is given by

$$
v_{1,2}=-\lambda^{2}=\frac{1}{2}\left[\left(4-\frac{3}{\sqrt{1-e^{2}}}\right) \pm \sqrt{\left.\left(4-\frac{3}{\sqrt{1-e^{2}}}\right)^{2}-\frac{27 \mu(1-\mu)}{1-e^{2}}\right]}\right.
$$

The above frequencies are obtained using the power series. The motion is periodic with periods $t_{1}=2 \pi / v_{1}$ and $t_{1}=2 \pi / v_{2}$. The short non-dimensional period of oscillation is $t_{1}=6.32$ and the longdimensional period of oscillation is $t_{2}=76.032$. The orbital period of Jupiter is $P=11.86$ years. The dimensional periods can be found as $T_{1}=P / \nu_{1}=11.932$ years and $T_{2}=P_{2} / \nu_{2}=143.54$ years [10]. The tadpole orbit is provided by the combination of long- and short- period motions around $\mathrm{L}_{4}$ from Eq. (29) for the Sun-Jupiter system for 30 revolutions. The two periods are also shown in Fig. (10). The loops are essentially due to the eccentricity of Jupiter.

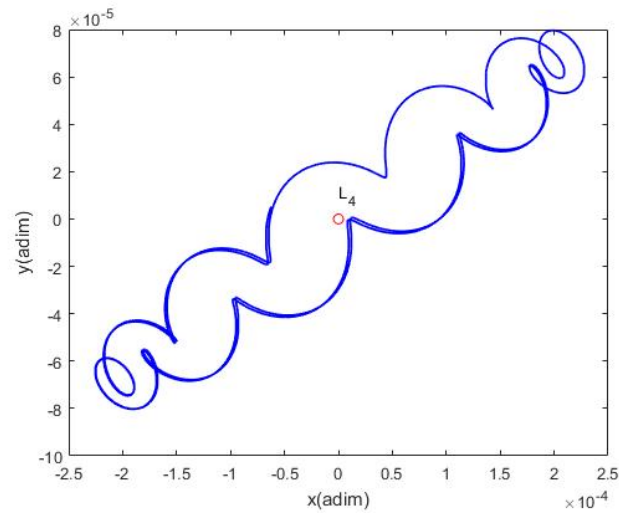

Figure 9. Tadpole orbit around $\mathrm{L}_{4}$ for Sun-Jupiter system

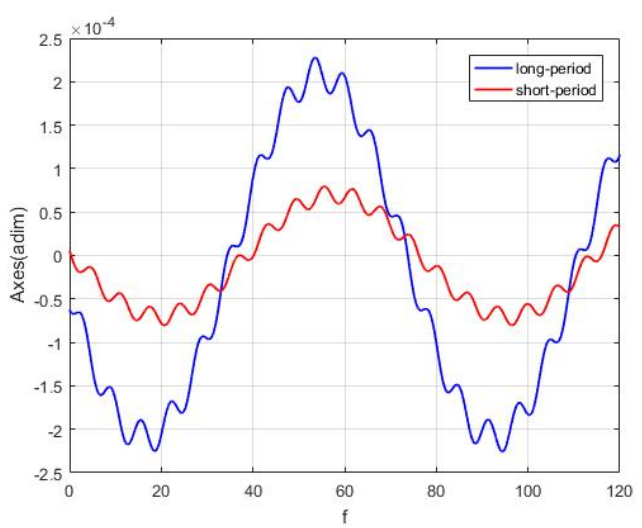

Figure 10. $f \in[0,40 \pi]$ with $\mathrm{x}-\mathrm{y}$ axes for combined long and short period motion in the Sun-Jupiter system 


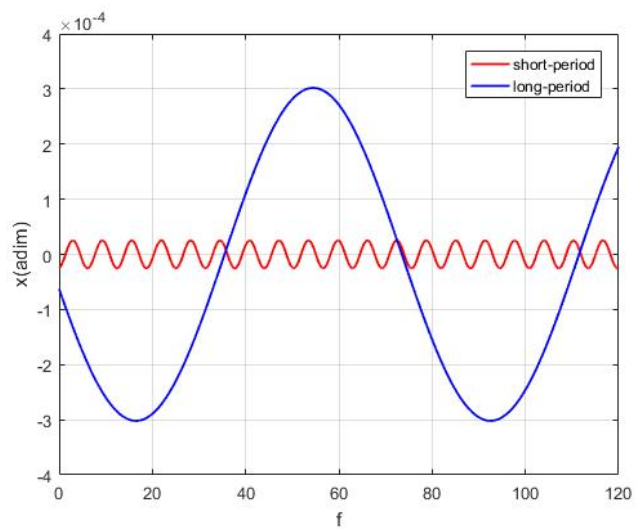

Figure 11. $f \in[0,40 \pi]$ with $\mathrm{x}$-axis for short and long period motions in the Sun-Jupiter system

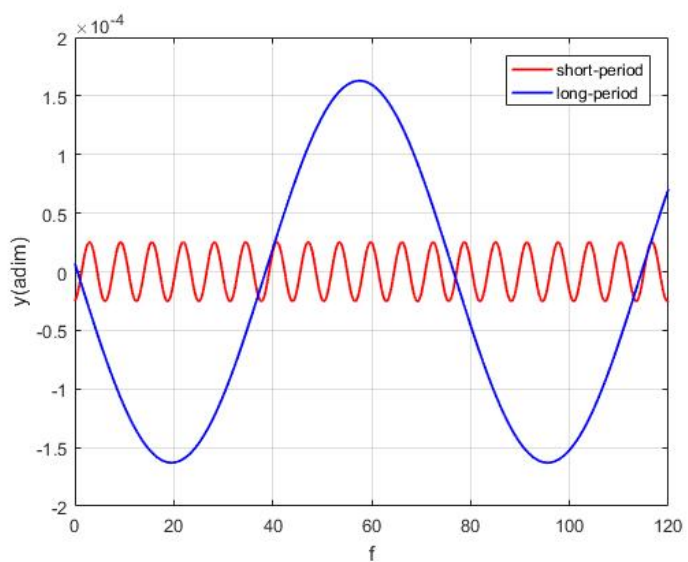

Figure 12. $f \in[0,40 \pi]$ with y-axes for short and long period motions in the Sun-Jupiter system

Motion around $\mathrm{L}_{4} / \mathrm{L}_{5}$ in the case of radiating and oblate primaries

When the perturbations of the primaries are considered, the potential function is

$$
\Omega=\frac{1}{2}\left(\varepsilon^{2}+\eta^{2}\right)+\frac{1}{n^{2}}\left[\frac{(1-\mu) q}{r_{1}}+\frac{\mu}{r_{2}}+\frac{\mu A_{2}}{2 r_{2}^{3}}\right]
$$

Differentiating the above equation, we get

$$
\begin{aligned}
& \Omega_{\varepsilon \varepsilon}=1+\frac{1}{n^{2}}\left(-\frac{(1-\mu) q}{r_{1}^{3}}+\frac{3(1-\mu) q(\varepsilon-\mu)^{2}}{r_{1}^{5}}-\frac{\mu}{r_{2}^{3}}-\frac{3 \mu(\varepsilon-\mu+1)^{2}}{2 r_{2}^{5}}-\frac{3 \mu A_{2}}{2 r_{2}^{5}}+\frac{15 \mu A_{2}(\varepsilon-\mu+1)^{2}}{2 r_{2}^{7}}\right) \\
& \Omega_{\eta \eta}=1+\frac{1}{n^{2}}\left(-\frac{(1-\mu) q}{r_{1}^{3}}+\frac{3(1-\mu) q \eta^{2}}{r_{1}^{5}}-\frac{\mu}{r_{2}^{3}}+\frac{3 \mu \eta^{2}}{r_{2}^{5}}-\frac{3 \mu A_{2}}{2 r_{2}^{5}}+\frac{15 \mu A_{2} \eta^{2}}{2 r_{2}^{7}}\right) \\
& \Omega_{\varepsilon \eta}=\frac{\eta}{n^{2}}\left(\frac{3(1-\mu)(\varepsilon-\mu) q}{r_{1}^{5}}+\frac{3 \mu(\varepsilon-\mu+1)}{r_{2}^{5}}+\frac{15 \mu A_{2}(\varepsilon-\mu+1)}{2 r_{2}^{7}}\right)
\end{aligned}
$$

Frequencies corresponding to the characteristic equation with the above values are

$$
v_{1,2}=-\lambda^{2}=\frac{1}{2}\left[\left(4-\Omega_{\xi \xi}-\Omega_{\eta \eta}\right) \pm \sqrt{\left(4-\Omega_{\xi \xi} \Omega_{\eta \eta}\right)^{2}-4\left(\Omega_{\xi \xi} \Omega_{\eta \eta}-\Omega_{\xi \eta}^{2}\right)}\right]
$$

Considering Sun-Saturn system where $\mu=0.000285635$ and $e=0.05386179$, when $\in=A_{2}=0$, the periods are $t_{1}=6.303$ and $t_{2}=148.4565$ from Eq. (29). The orbital period of Saturn is $P=29.45$ years. Hence the dimensional periods are $T_{1}=29.4519$ years and $T_{2}=657.50$ years. Tadpole orbit developed about the $\mathrm{L}_{4}$ 
point in this system for 30 revolutions is shown in Fig. (14). With the effect of radiation pressure, the non-dimensional periods are $t_{1}=6.343$ and $t_{2}=142.456$ from Eq. (32). The dimensional periods can be calculated as $T_{1}=29.387$ years and $T_{2}=634.66$ years. The orbit with the influence of radiation for 60 revolutions is shown in Fig. (15).

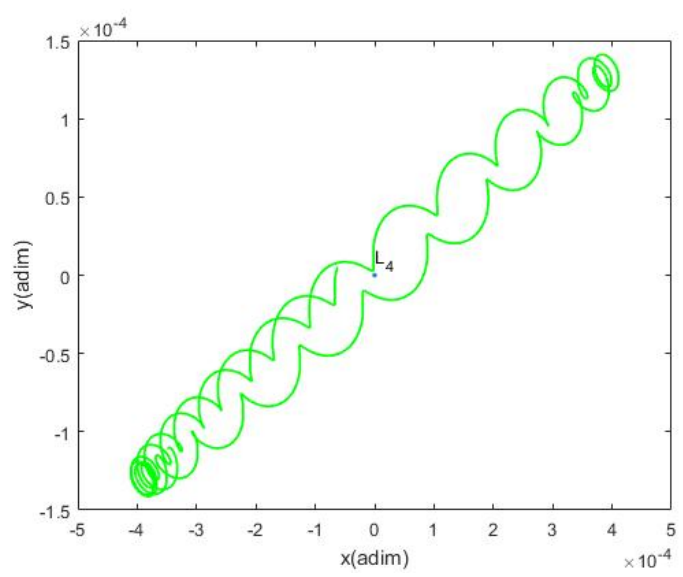

Figure 13. Motion around $L_{4}$ for Sun-Saturn system when $\epsilon=A_{2}=0$.

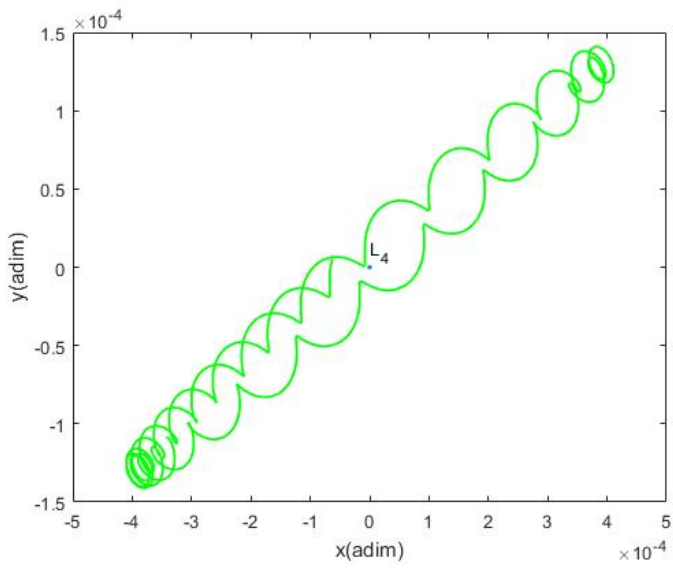

Figure 14. Motion around $\mathrm{L}_{4}$ for Sun-Saturn system for 30 revolutions

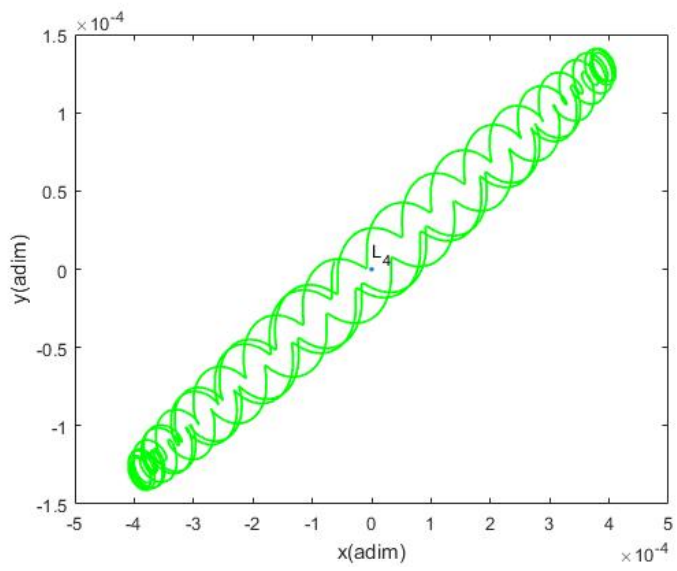

Figure 15. Motion around $\mathrm{L}_{4}$ for Sun-Saturn system for 60 revolutions 


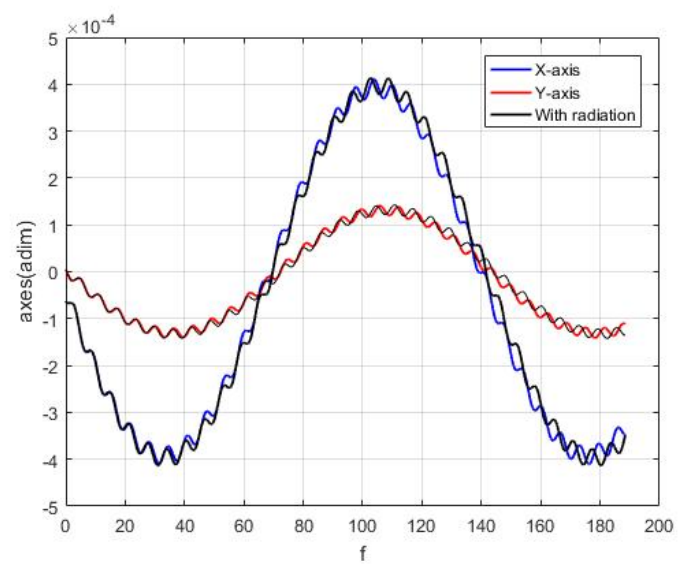

Figure 16. $f \in[0,60 \pi]$ with $x-y$ axes for combined long and short periodic orbits in the case of Sun-Saturn system with and without radiation.

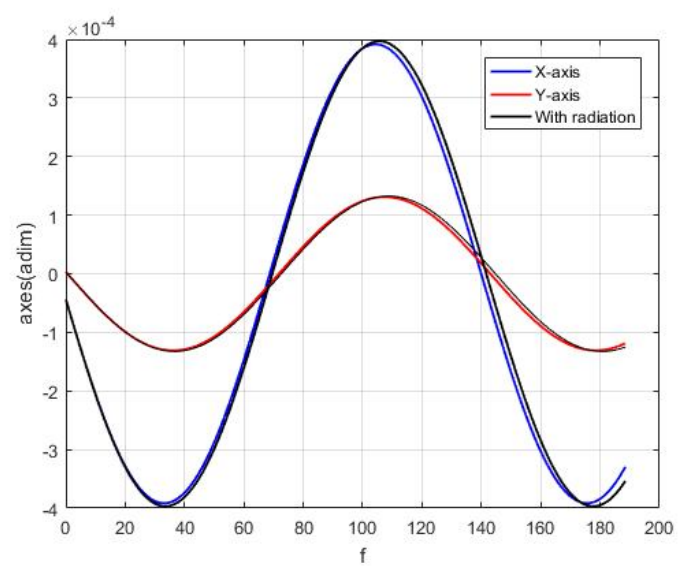

Figure 17. $f \in[0,60 \pi]$ with $\mathrm{x}$ and $\mathrm{y}$ axes for long periodic orbits in the case of Sun-Saturn system with and without radiation.

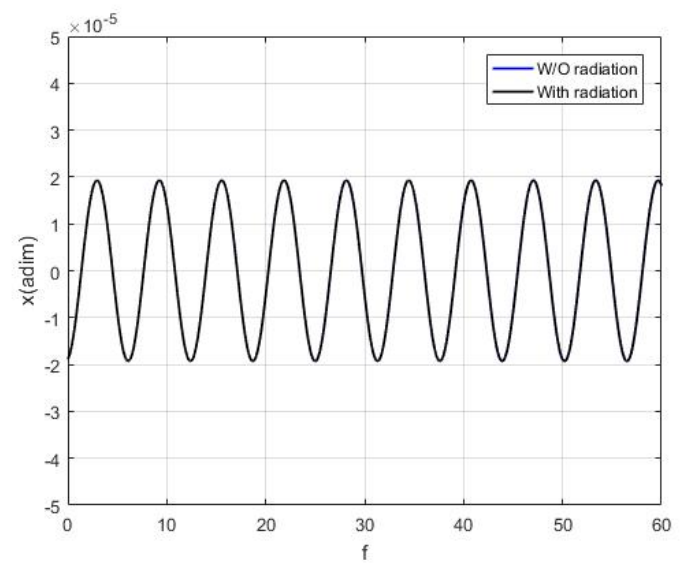

Figure 18. $f \in[0,60 \pi]$ with x-axes for short periodic orbit in the case of Sun-Saturn system with and without radiation. 


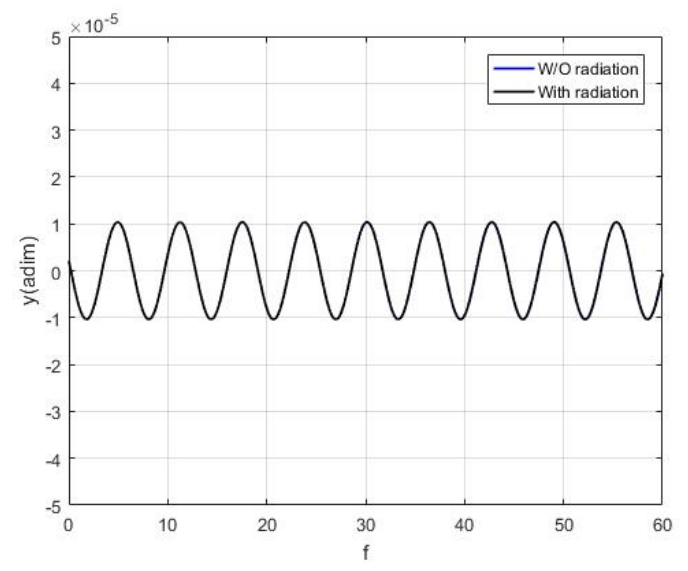

Figure 19. $f \in[0,60 \pi]$ with y-axes for short periodic orbits in the case of Sun-Saturn system with and without radiation.

\section{$7 \quad$ Results and Conclusion}

The mean motion ' $n$ ' is derived by averaging the distance ' $r$ ' between the primaries over a revolution in terms of the mean anomaly in the photogravitational elliptic restricted three-body problem when the smaller primary is an oblate spheroid with its equatorial plane coincident with the plane of motion. The value of $n$ is different from the mean motion derived using the true anomaly for averaging the distance ' $r$ ' in the literature. The locations of $\mathrm{L}_{1}, \mathrm{~L}_{2}, \mathrm{~L}_{3}$ and their stability are studied. Significant effect is found on the locations of these points. The points $\mathrm{L}_{1}, \mathrm{~L}_{2}, \mathrm{~L}_{3}$ move away from the larger primary with the inclusion of radiation pressure. The critical value of mass parameter $\mu_{c}$ is obtained. It decreases with the increase in radiation pressure and oblateness. This critical value is used to determine the size of the region of stability. The curves separate the stable region from the unstable region. The stability of the triangular points decreases with the increase in radiation pressure and oblateness. The zone of stability shrinks when the radiation pressure increases. The tadpole orbits for Sun-Jupiter system with distinguished long- and short- periodic orbits for 30 revolutions are plotted. The periods of the shortlong periodic orbits show a change from the relative circular problem for the same model. While the short-period does not show much deviation, it indicates significant influence of the Jupiter's eccentricity in the librational motion of tadpole orbit. The epicyclic oscillation of the tadpole orbit still does not deviate much because of the very low value of oblateness coefficient ' $A_{2}{ }^{\prime}$.

Acknowledgements. The authors are thankful to the referee for the comments and suggestions which enabled the presentation in the present form.

\section{References}

1. Danby, (1964). "Stability of the Triangular Points in the Elliptic Restricted Problem", The Astronomical Journal, 69, No. 2

2. R.K.Sharma (1987). "The Linear stability of Libration Points of the Photogravitational Restricted Three-Body Problem when the smaller primary is an oblate spheroid", Astrophysics and Space Sciences 135 (1987) 271-281.

3. S.K.Sahoo and B.Ishwar (2000). "Stability of collinear equilibrium points in the generalized photogravitational elliptic restricted three-body problem". Astronomical Society of India,28, 579-586.

4. J. Singh, A. Umar (2012). "On out of plane equilibrium points in the ER3BP with radiating and oblate primaries". Astrophys, Space Science, 344:13-19.

5. P.V. Subba Rao and R.K. Sharma (1975). "A note on the Stability of the Triangular Points of the Equilibrium in the Restricted Three-body Problem". Astron $\&$ Astrophys. 43, 381-383.

6. R.K.Sharma and P.V.Subba Rao, "Stationary solutions and their characteristic exponents in the restricted threebody problem when the more massive primary is an oblate spheroid". Celestial Mechanics 13 (1976) 137-149. 
7. E.A.Grebenikov, "On the Stability of The Lagrangian Triangle Solutions of the Restricted Elliptic Three Body Problem," Soviet Astronomy- AJ, 1964, vol 8, No.3.

8. C. D. Murray and S. F. Dermott. (1999). "Solar System Dynamics", Cambridge University Press, Cambridge.

9. R. A. Broucke (1969), "Periodic orbits in the Elliptic Restricted Three-body problem". Technical Report 32-1360.

10. Szebehely, V.:1967, Theory of Orbits, "The Restricted Problem of Three bodies", Academic Press, New York.

11. R. A. Broucke, "Stability of Periodic Orbits in the Elliptic Restricted Three-body Problem", Paper 68-086, presented at the AAS/AIAA Astrodynamics Specialist Conference, Jackson, Wyo., Sept- 3-5,1968.

12. K. T. Alfriend and R. H. Rand. (1969)." Stability of the Triangular Points in the Elliptic Restricted Problem of Three bodies", AIAA Journal, 7, No 6, 1024-1028.

13. V. Kumar, R. K. Choudhary, Non linear stability of the triangular libration points for the photogravitational elliptic restricted problem of three bodies, Celestial Mech. and Dyn. Astro.,48, No. 4(1990), 299-317.

14. V. V. Markellos, E. Perdios and P. Labropoulou (1992)."Linear stability of the triangular equilibrium points in the photogravitational elliptic restricted problem I", Astrophysics and Space Science 194, 207-213.

15. Raheem \& Singh. (2006). "Combined effects of perturbations, radiation, and oblateness on the stability of equilibrium points in the restricted three-body problem." The Astronomical Journal, 131:1880-1885, 2006 March.

16. A. Narayan and C. R. Kumar. (2011). "Stability of Triangular Equilibrium points in elliptical restricted threebody problem under the effects of photogravitational and oblateness of primaries", International Journal of Pure and Applied Mathematics, Volume 70 No. 5 2011, 735-754.

17. A. Bennett. (1965), "Analytical determination of characteristic exponents", The Boeing Company Huntsville, Alabama, AIAA Paper, NO. 65-685.

18. A. Narayan and N.Singh (2015), "Characteristics Exponents of the Triangular Solutions in the Elliptical Restricted Three-Body Problem under Radiating Primaries", Differ Equ Dyn Syst 24(3):329--343.

19. R. Rajnai, L. Nagy and B. Erdi. (2002), "Frequencies and resonances around $\mathrm{L}_{4}$ in the elliptic restricted threebody problem", Mon. Not. R. Astron. Soc. 000, 1-10.

20. P. Gurfil, D. Meltzer (2006), "Stationkeeping on unstable orbits: Generalization to the elliptic restricted threebody problem", The Journal of the Astronautical Sciences, Vol. 54, 29-51.

21. J. Singh and A. Umar (2014). "The collinear libration points in the elliptic restricted three-body problem with a triaxial primary and an oblate secondary", Astronomy and Astrophysics 4, 391-398.

22. A. G. Anderlecht, "Tadpole orbits in the $\mathrm{L}_{4} / \mathrm{L}_{5}$ region: Construction and links to other families of periodic orbits", Thesis (2016), Purdue University, West Lafayette, Indiana.

23. E.I. Abouelmagd et al. (2016) "Periodic orbits around the collinear libration points", Journal of Nonlinear Science and Applications, 9, 1716-172\%.

24. V. V. Radzievsky (1950). "The restricted problem of three bodies taking account of light pressure", Astron Zh, 27, $p$. 250 .

25. J. H. Poynting (1903) "The pressure of light, The Inquirer", pp. 195-196.

26. J. M. A. Danby., (1988), "Fundamentals of Celestial Mechanics", (pg 346).

27. S. Kumar, S. and B. Ishwar (2009), "Solutions of Generalized Photogravitational Elliptic Restricted Three-Body Problem." AIP Conference Proceedings, 1146, 456-460. 